\title{
Implementation of Home based management of malaria in children reduces the work load for peripheral health facilities in a rural district of Burkina Faso
}

\author{
Alfred B Tiono ${ }^{1}$, Youssouf Kaboré ${ }^{1}$, Abdoulaye Traoré ${ }^{1}$, Nathalie Convelbo ${ }^{1}$, \\ Franco Pagnoni ${ }^{3}$ and Sodiomon B Sirima*1,2
}

Address: ${ }^{1}$ Centre National de Recherche et de Formation sur le Paludisme, Ministère de la Santé, Ouagadougou, Burkina Faso, West Africa, ${ }^{2}$ Groupe de Recherche et d'Action en Santé (GRAS), Ouagadougou, Burkina Faso, West Africa and ${ }^{3}$ UNICEF/UNDP/World Bank/WHO Special Programme for Research and Training in Tropical Diseases, World Health Organization, Geneva, Switzerland

Email: Alfred B Tiono - t.alfred@fasonet.bf; Youssouf Kaboré - kabyouf@yahoo.fr; Abdoulaye Traoré - traorabdoulay@yahoo.fr; Nathalie Convelbo - nath_convelbo@ hotmail.com; Franco Pagnoni - pagnonif@who.int; Sodiomon B Sirima* - s.sirima.cnlp@fasonet.bf

* Corresponding author

Published: 3 October 2008

Malaria Journal 2008, 7:20I doi:10.1/86/1475-2875-7-20I
Received: II May 2008

Accepted: 3 October 2008

This article is available from: http://www.malariajournal.com/content/7/I/20I

(c) 2008 Tiono et al; licensee BioMed Central Ltd.

This is an Open Access article distributed under the terms of the Creative Commons Attribution License (http://creativecommons.org/licenses/by/2.0), which permits unrestricted use, distribution, and reproduction in any medium, provided the original work is properly cited.

\begin{abstract}
Background: Home Management of Malaria (HMM) is one of the key strategies to reduce the burden of malaria for vulnerable population in endemic countries. It is based on the evidence that well-trained communities health workers can provide prompt and adequate care to patients close to their homes. The strategy has been shown to reduce malaria mortality and severe morbidity and has been adopted by the World Health Organization as a cornerstone of malaria control in Africa. However, the potential fall-out of this community-based strategy on the work burden at the peripheral health facilities level has never been investigated.
\end{abstract}

Methods: A two-arm interventional study was conducted in a rural health district of Burkina Faso. The HMM strategy has been implemented in seven community clinics catchment's area (intervention arm). For the other seven community clinics in the control arm, no HMM intervention was implemented. In each of the study arms, presumptive treatment was provided for episodes of fevers/malaria (defined operationally as malaria).

The study drug was artemether-lumefantrine, which was sold at a subsidized price by community health workers/Key opinion leaders at the community level and by the pharmacists at the health facility level.

The outcome measured was the proportion of malaria cases among all health facility attendance (all causes diseases) in both arms throughout the high transmission season.

Results: A total of 7,62I children were enrolled in the intervention arm and 7,605 in the control arm. During the study period, the proportions of malaria cases among all health facility attendance (all causes diseases) were $21.0 \%,(445 / 2,1 \mathrm{II}, 95 \% \mathrm{Cl}$ [ $19.3 \%$ $22.7 \%])$ and $70.7 \%(2,595 / 3,67 \mathrm{I}, 95 \% \mathrm{Cl} 68.5 \%-7 \mathrm{I} .5 \%)$, respectively in the intervention and control arms ( $\mathrm{p}<<0.000 \mathrm{I})$. The relative risk ratio for a fever/malaria episode to be treated at the $\mathrm{HF}$ level was $30 \%(0.30<R R<0.32)$.

The number of malaria episodes treated in the intervention arm was much higher than in the control arm $(6,66 \mathrm{I}$ vs. 2,595), with malaria accounting for $87.4 \%$ of all disease episodes recorded in the intervention area and for $34.1 \%$ in the control area $(P<$ 0.000 I). Of all the malaria cases treated in the intervention arm, only $6.7 \%$ were treated at the health facility level.

Conclusion: These findings suggest that implementation of HMM, by reducing the workload in health facilities, might contributes to an overall increase of the performance of the peripheral health facilities. 


\section{Background}

Malaria remains the leading cause of child mortality and morbidity in sub-Saharan Africa [1-3]. High malaria transmission intensity, limited access to adequate treatment, increasing parasite resistance to affordable and safe medicines (chloroquine, sulphadoxine-pyrimethamine and amodiaquine); increasing vectors resistance to widely used insecticides, delayed care-seeking and inappropriate treatment at home or at community level, are some of the causes for this deleterious situation.

In 2000, the African Heads of State adopted the access to prompt and effective treatment for at least $60 \%$ of those suffering from malaria as one of the goal in reducing the malaria burden for the vulnerable population in Africa [4]. This goal in the context of the limited coverage of the population by health facilities in most of the malaria endemic countries seems quite ambitious and difficult to achieve. Therefore, the Home Management of Malaria (HMM) strategy that has been developed to provide prompt and effective treatment of malaria episodes to those who need it close to their homes represents one of the major strategies that could contribute to achieve this goal [5].

The Roll Back Malaria (RBM) initiative has defined the HMM as an integral part of an overall malaria case management strategy aiming to improve access to treatment for malaria in areas with limited access to health facilities. The strategy aims to improve the ineffective self-medication practices that are very common in malaria-endemic countries; its overall goal is the early recognition and prompt and appropriate response to malarial illness, especially in children under five years of age, in the home or community. It, therefore, empowers communities to respond to malaria illness using effective, good-quality antimalarial medicines through community involvement [5].

Several studies conducted under the leadership of the World Health Organization, have demonstrated its feasibility and efficacy [6-8]. In recent years, with the almost universal development of CQ resistance [9-11], HMM is being promoted with artemisinin-based combination therapy (ACT). Despite some concerns on the widespread use of such drugs at community level [12] recent data have shown that ACT can be used safely within the context of the HMM strategy $[13,14]$.

In Burkina Faso, the HMM strategy has been adopted by the National Malaria Control Programme in an effort to reach the Abuja target. Unfortunately, artemether-lumefantrine (AL) (Coartem $^{\circledast}$ ), which is to be used at the community level in the context of this strategy, is not yet available in the public sector; the implementation of the strategy is, therefore, facing some delays.
During the high transmission season of malaria, episodes of fever/malaria (from now on operationally defined as 'malaria') in children, account for more than $1 / 3$ of the attendances at first level health facilities. A limited number of health personnel in peripheral health facilities named Centre de Santé et de Promotion Social (CSPS) (usually no more than three staff members including two nurses and one midwife) are assigned to perform a minimal package of activities, including antenatal care services, out-patient management, Expanded Programme of Immunization (EPI), counselling, administrative tasks etc. This results in a very high workload with a potential negative impact on the quality of the services provided.

This study has evaluated the effect of the case management of malaria at a more peripheral level than the health facility - in the community within the context of HMM on the workload of the health staff operating in first level health facilities.

\section{Methods}

The study was an interventional study, conducted in the rural health district of Saponé located at around $45 \mathrm{~km}$ south of Ouagadougou, the capital of Burkina Faso. The climate in the area is characteristic of the Sudanese savannah, with a dry season from November to May and a rainy season from June to October. Malaria transmission is markedly seasonal, with most transmission occurring during the rainy season with an entomological inoculation rate ranging from 50-200 infective bites/person/year. Plasmodium falciparum is the most predominant malaria parasite accounting for more than $95 \%$ of infections in children less than five years of age. Malaria burden is heaviest among children aged less than 5 years, with every child experiencing an average of two clinical malaria episodes every transmission season.

The health district area covers fourteen community clinics (CSPS), which represent the first level of care in the health system of Burkina Faso. The mean area covered by the catchment area of each CSPS is $9.7 \mathrm{~km}^{2}$. Thus, the population has access to the CSPS within an average walking distance of $10 \mathrm{~km}$.

There is no private practice of medicine and drugs selling in the area. Population has legally access to treatment drugs (exclusively generics) including antimalarial drugs through the drug store located in these community clinics; however, there is a parallel market where many other illegally imported drugs are sold.

The study was not randomized. The fourteen community clinics were equally assigned to one of the study arms: seven in the intervention arm and seven in the control arm. Prior to any activity, discussion was conducted with 
the communities to inform them about the research and seek their assent to participate.

A baseline general census was afterward conducted in both arms to determine the population size, with main focus on the target population for the intervention (children less than five years of age). A health care-seeking behaviour study was conducted to identify, at baseline, whether the two study arms were comparable in regard of this aspect.

Following these baseline activities, in the intervention arm, Community Health Workers (CHWs) and Key Opinion Leaders (KOLs) were identified by their respective communities and trained by the study team to recognize the symptoms of uncomplicated malaria and provide the study treatment, which was artemether-lumefantrine $(\mathrm{AL})\left(\mathrm{Coartem}^{\circledast}\right)$. AL was pre-packaged in two different blister packs by the manufacturer for children aged between 6-35 months (yellow blister pack) and 36-60 months (blue blister pack). CHWs/KOLs were also trained in recognition of symptoms of severity of malaria (prostration, coma, convulsions), as well as other childhood non-malaria diseases, such as pneumonia (cough with fast breathing) or acute diarrhoea, which require immediate referral to the nearest health facility.

In terms of role and responsibilities, while the CHWs and the KOLs were both sharing the responsibility of providing treatment to the sick children, the CHW was playing the additional role of local suppliers of the study drugs to a certain number of KOLs under his/her responsibility. They were responsible for getting the study drugs at the health facility drug store, for their own stock renewal and for supplying the KOLs under their coverage. Thus, CHWs were acting as the link between the local health facility and the KOLs in terms of study drugs supply chain.

The nurses and the drug-store keepers at the health facility have assisted the study team in the training of the CHWs/ KOLs. The training has included $189 \mathrm{CHWs} /$ KOLs in the intervention arm. For both arms, 30 nurses (15 in each arm) and 14 drug-stores keepers (7 in each arm) were trained. A post-training survey was conducted to assess CHWs/KOLs knowledge, attitude and practices in order to identify any need of retraining before the study start. An oral interview was conducted with a sample of 40 CHWs and 127 KOLs, of which 56 males (33.5\%) and 111 females (66.5\%). A questionnaire was used.

The mean age was 33.5 years (range 16 to 60 ). The majority were farmers $(98.8 \%)$.

Most of the CHWs/KOLs were able to recognize the malaria symptoms (Table 1). In particular, the main symptom (hot body) was mentioned by the totality of the interviewees.

The knowledge of the CHWs/KOLs on the requirements for the study drug prescription was also assessed taking the example of the case of a five-months old child; a wrong prescription was noted for 8 CHWs/KOLs (4.2\%); the study drug was not indicated for the majority.

Criteria of severity like pallor, fast breathing and convulsions warranting urgent referral were also mentioned by all the workers. At the end of the evaluation session, the need of refresher training on the study drugs prescription was identified. All the CHWs/KOLs benefited from this training.

Table I: Baseline characteristics

\begin{tabular}{|c|c|c|c|}
\hline & $\begin{array}{l}\text { Intervention arm } \\
(\mathbf{N}=1021)\end{array}$ & $\begin{array}{l}\text { Control arm } \\
(N=1034)\end{array}$ & $\mathbf{P}$ \\
\hline Mean age of the caregivers (mean $\pm \mathrm{SD}$; range) & $24.3 \pm 6.3(17 ; 52)$ & $23.6 \pm 5.8(18 ; 45)$ & 0.14 \\
\hline Number of children less than five years (mean \pm SD; range) & $\begin{array}{l}1.5 \pm 0.01 \\
\quad(1 ; 3)\end{array}$ & $\begin{array}{l}1.4 \pm 0.01 \\
\quad(1 ; 3)\end{array}$ & 0.21 \\
\hline \multicolumn{4}{|l|}{ Knowledge of malaria transmission } \\
\hline Mosquitoes & $282(27.6 \%)$ & $291(28.1 \%)$ & 0.79 \\
\hline Others (rain, sun) & $138(13.5 \%)$ & $112(10.8 \%)$ & 0.06 \\
\hline \multicolumn{4}{|c|}{ Health care-seeking behaviour (first option for treating malaria episodes) } \\
\hline Use of health facility & $289(28.3 \%)$ & $278(26.9 \%)$ & 0.45 \\
\hline Self-treatment & $732(71.7 \%)$ & $758(73.3 \%)$ & 0.45 \\
\hline \multicolumn{4}{|l|}{ Children malaria symptoms mentioned by the caregivers } \\
\hline Hot body & 748 (73.3\%) & $752(72.7 \%)$ & 0.78 \\
\hline Crying more than usual & 161 (15.8\%) & $186(18.0 \%)$ & 0.18 \\
\hline Yellowish vomiting & $358(35.1 \%)$ & 385 (37.2\%) & 0.30 \\
\hline Anorexia & $229(22.4 \%)$ & $190(18.4 \%)$ & 0.02 \\
\hline Others & $39(3.8 \%)$ & $43(4.2 \%)$ & 0.69 \\
\hline
\end{tabular}


A study drug (AL) distribution channel was set up. The study drugs were made available by the project to the district main drug store, which supplied each community clinic drug-store. The community clinic drug-store in turn supplied the CHWs acting in their respective villages as main drug-store. The KOLs in each village were supplied by their respective CHWs. For a self-sustainability of the distribution channel, an incentive margin of 10 FCFA (0.024 USD) (per unit of treatment sold) was allowed at each step of the distribution channel.

To initiate the distribution channel, each level was provided proportionally to its need with a seed stock of $\mathrm{AL}$, made available free-of-charge by the project. Final cost of the drug for the caregivers was 100 FCFA (0.24 USD) for the yellow blister pack and 200 FCFA (0.48 USD) for the blue blister pack, whatever place they purchased it (at the $\mathrm{CHWs} / \mathrm{KOLs}$ level or at the community clinic drug-store level). The standard price of Coartem ${ }^{\circledast}$ was 10 USD in the private sector. AL was not yet available in the public sector at the time of the study.

Despite an effort to bring down the cost of drugs in order to improve as much as possible the financial access for the caregivers, it was anticipated that some vulnerable groups may not be able to afford the cost for the drugs. The CHWs and the KOLs were instructed to deliver the drugs free-ofcharge to such groups. The cost was reimbursed to them by the project.

To enhance accessibility of caregivers to CHWs and, therefore, their adherence to the strategy, a ratio of one CHW/ KOL per 40 children was adopted. Taking into account the population density of the study area, this ratio offers the advantage that any sick child will be living within less than 25 minutes walk from a CHW/KOLs household.

In the control arm, there were no CHWs/KOLs at the community level. AL was made available in both arms at the health facilities level, at the same cost as in the community [100 Francs CFA (0.24 USD) for the yellow blister pack and 200 Francs CFA (0.48 USD) for the blue blister pack].

The intervention has started in May 2006 and the study drugs were deployed in July 2006. A survey was conducted in October 2006 (at the end of malaria high transmission season) both at community and health facility level to assess activities in terms of malaria cases management of CHWs/KOLs and health centre staff.

\section{Statistical analysis}

Paper forms were used to collect the study data. The completed questionnaires were verified and data double entered electronically and analysed using EPI-INFO (Ver- sion 6.04) (CDC, Atlanta, USA and WHO). Standard quality control checks were instituted to ensure the consistency of the data.

The primary outcome to be measured was the number of cases treated for malaria in both study arms. Malaria episodes were defined as the caregiver reporting to the $\mathrm{CHWs} / \mathrm{KOLs}$ or to the health facility because of his/her child experiencing/having experienced an episode of "hot $b o d y^{\prime \prime}$. No measurement of temperature or of malaria parasitaemia was performed. The use of a case definition based on "hot body" is in line with the WHO HMM strategy and has been previously adopted by Sirima et al [4]. From the national health statistics, malaria represent on average $30 \%$ of all causes health facility attendance; the minimum sample size required was, therefore, 263 malaria cases treated per study arm over the three months period of the study to detect a reduction of at least $25 \%$ between the intervention and the control arm (with 95\% confidence interval).

The impact of the intervention in reducing the health facility attendance for malaria episodes in the study population has been calculated using the formula 1 minus the rate ratio (relative risk). Pearson's chi test was used to compare proportions at the significance level of $\mathrm{P}=0.05$.

\section{Ethical clearance}

The study protocol was reviewed and approved by the ethics committee of the WHO and the National Ethical Committee of Burkina Faso. Appropriate authorities in the district were informed and approval sought from them. Community meetings were held with community leaders in the intervention arm, to discuss with them the purpose of the study and to obtain their adhesion.

\section{Results}

\section{Background information}

The baseline census showed a total population of 40,649 in the intervention arm and 40,557 in the control arm. Children aged less than five years were 7,621 and 7,605 respectively. The study profile is provided is figure 1 .

To check if there was any difference in health-seeking behaviour in the two arms, a baseline survey was conducted randomly in 1,021 mothers/caregivers in the intervention arm and 1,034 in the control arm. The use of health facilities for the treatment of children fevers was declared as the first option for $28.3 \%$ and $26.9 \%$, respectively in the intervention and the control arms $(\mathrm{p}=0.45)$. In terms of clinical signs/symptoms of malaria, the hot body was reported by most of the caregivers $(73.3 \%$ in intervention arm versus $72.7 \%$ in the control arm ( $\mathrm{p}=$ 0.78 ). Other signs reported were yellowish vomiting, crying more than usual and anorexia (Table 1). 


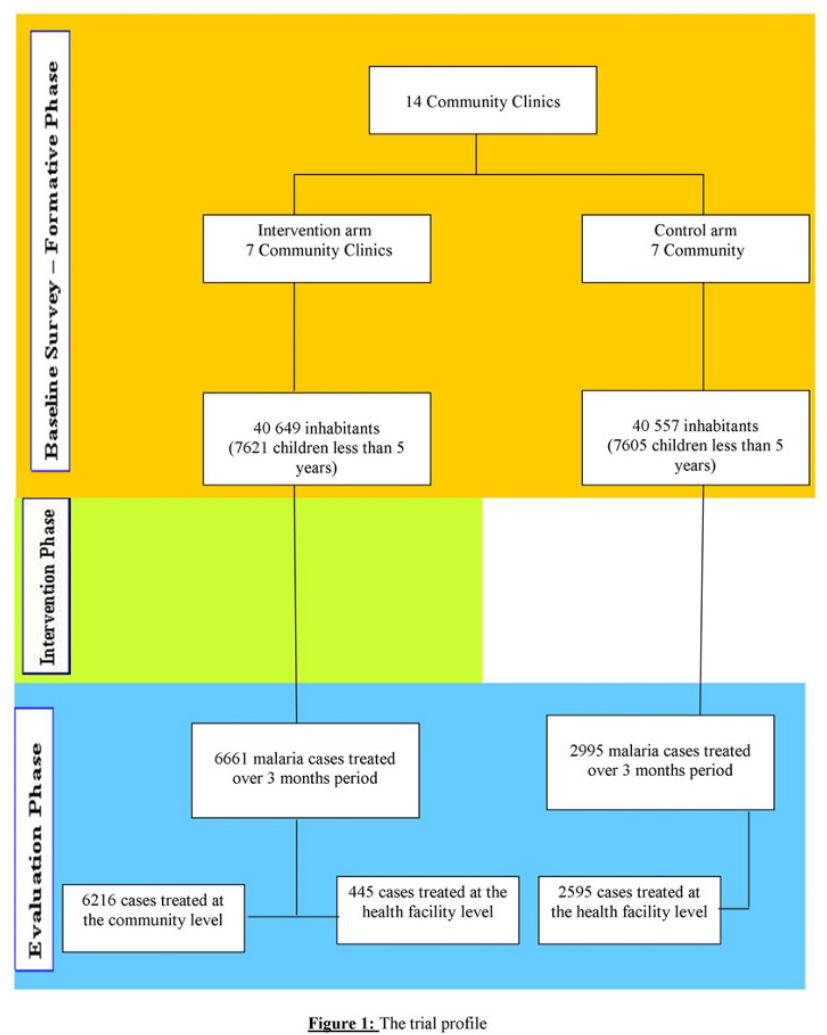

Figure I

The trial profile.

\section{Episodes of disease (all causes) treated at health facility (HF) level}

From August to October 2006, 2,111 episodes of disease (all causes) in children under the age of five were treated in HF of the intervention arm, and 3,671 in HF of the control arm; this corresponds to an incidence of disease episodes of $27.7 \%(2,111 / 7,621)$ and $48.3 \%(3,671 / 7605)$, respectively (95\% CI $26.7 \%-28.7 \%$ and $47.2 \%-49.4 \%$ ). Within each of the study arms, there was a trend to an increase of the incidence of disease episodes over the three-month study period (Table 2). The overall workload in the HF of the intervention area was thus reduced by $43 \%, \mathrm{p}<<0.0001$.
Non-malaria diseases treated at the health facility level have represented $29.3 \%$ in the control arm $(1,076 / 3,671)$ versus $78.9 \%(1,666 / 2,111)$ in the intervention arm $(\mathrm{P}<<$ 0.0001). (Figure 2)

\section{Episodes of malaria treated at $\mathbf{H F}$ and community level} A total of 6,661 malaria cases were treated in the intervention arm, where HMM was available, versus 2,595 in the control arm, where treatment was available only at HF level. Thus, the malaria episodes accounted for $87.4 \%$ of all disease episodes recorded in the intervention area and for $34.1 \%$ in the control area $(P<0.0001)$. Of all the malaria cases treated in the intervention arm, only 445 $(6.7 \%)$ were treated at the health facility level. Thus, almost $90 \%$ of the malaria cases in this arm have been treated by the CHWs/KOLs at the community level.

In general, for the population of children aged less than five years, health facility attendance for malaria episodes has represented $21.0 \%$ of overall $\mathrm{HF}$ attendance in the intervention arm and $70.7 \%$ in the control arm (445/ $2,111,95 \%$ CI $19.3 \%-22.7 \%$ and $2,595 / 3,671,95 \%$ CI $68.5 \%-71.5 \%, \mathrm{p}<0.0001)$ (Figure 1). This translates in a relative risk ratio of $30 \%(0.30<\mathrm{RR}<0.32)$ of a malaria episode to be treated at HF level in the intervention arm. The impact of the intervention in reducing the proportion of malaria episodes of the total number of cases managed at the health facility level was, therefore, estimated to be $70.0 \%$.

\section{Discussion}

The outcome measurement has occurred within a relatively short time period after the intervention phase of the study (three months). This period, which corresponds to the end of the high transmission season, when all the players (HF, CHWs/KOLs) have gone through intense activities, is considered as the most accurate to detect significant impact of the intervention on the workload imputable to malaria cases management. A similar time window was used by Sirima et al [8]. Question on whether the findings of this study will be observed if the outcome measurement was done after a longer period is to be considered; however, in the context of the study area, where the malaria transmission is highly seasonal, the HMM

Table 2: Incidence of the diseases episodes at the health facility level

\begin{tabular}{|c|c|c|c|c|c|c|}
\hline \multirow[b]{2}{*}{ Months } & \multicolumn{3}{|c|}{ Intervention arm (N = 762I) } & \multicolumn{3}{|c|}{ Control arm $(\mathrm{N}=7605)$} \\
\hline & $\begin{array}{c}\text { Nb. of diseases episodes } \\
n\end{array}$ & $\begin{array}{l}\text { Incidence } \\
n / N\end{array}$ & $95 \%$ ci & $\begin{array}{c}\text { Nb. of diseases episodes } \\
n\end{array}$ & $\begin{array}{l}\text { Incidence } \\
n / N\end{array}$ & $95 \%$ ci \\
\hline August & 526 & $6.9 \%$ & $(6.3 \%-7.5 \%)$ & 1009 & $13.3 \%$ & $(12.5 \%-14.1 \%)$ \\
\hline September & 575 & $7.5 \%$ & $(6.9 \%-8.1 \%)$ & 1182 & $15.5 \%$ & $(14.7 \%-16.3 \%)$ \\
\hline October & 1010 & $13.3 \%$ & $(|2.5 \%-| 4.1 \%)$ & 1480 & $19.5 \%$ & (18.6\%-20.4\%) \\
\hline Total & 2111 & $27.7 \%$ & $(26.7 \%-28.7 \%)$ & 3671 & $48.3 \%$ & $(47.2 \%-49.4 \%)$ \\
\hline
\end{tabular}




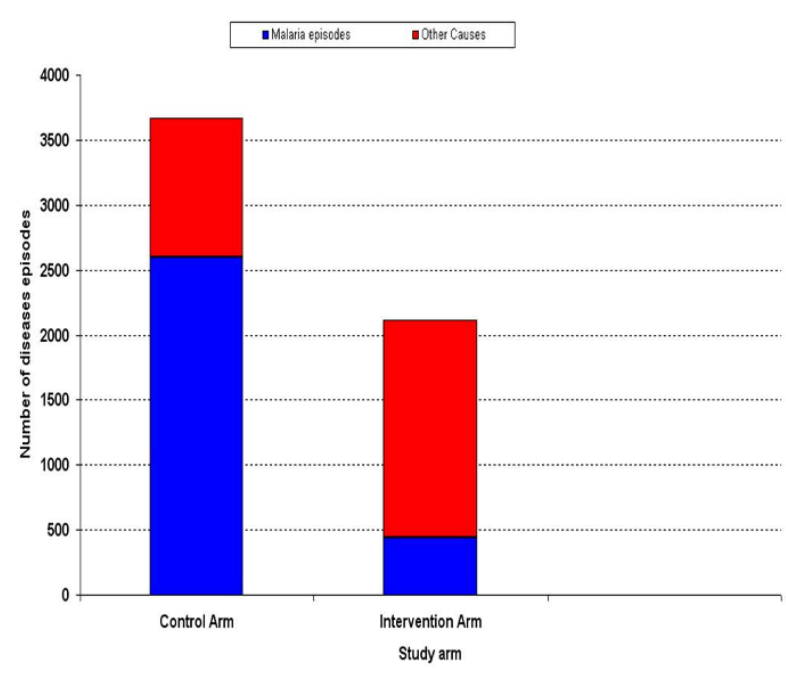

Figure 2: Number of diseases episodes (all causes) and malaria episodes treated at the health facility level

\section{Figure 2 \\ Number of diseases episodes (all causes) and malaria episodes treated at the health facility level.}

strategy will be implemented within the same timeframes used in this study to avoid wastage of resources and minimize the over-treatment of malaria during the low transmission season, when prevalence of the disease is very low. Therefore, the findings of this study could be considered as valid for areas with the same transmission pattern; different assessment timeframe need to be implemented in areas with a longer transmission period, to account for the necessity to sustain the HMM strategy in order to induce an optimum impact on the outcome measured in this study.

The number of malaria episodes treated in the intervention arm was more than double the number of cases treated in the control arm $(+156 \%)$. This shows that once the option of treating malaria close to home is made available, caregivers make use of it and the number of malaria episodes treated increases. No other reason can explain this finding, as both areas have a similar epidemiological profile and malaria transmission pattern, with comparable health care-seeking behaviour, as shown in the baseline data. The most plausible explanation is thus that the presence of CHWs/KOLs, by increasing the access to an effective treatment in the intervention arm, has boosted the caregiver's willingness to use this new possibility, reducing the use of other sources of treatment.

The presence of the CHWs/KOLs is also the possible explanation to the fact that a significant difference was observed between intervention and control arms in terms of the treatment of non-malaria diseases. Indeed, while a reduction of number of malaria cases treated at the health facility level was observed in the intervention arm, the proportion of non-malaria cases treated there was significantly higher as compared to the control group.

It is possible that, at the community level, the CHWs/ KOLs have played the role of "triage staff" for the health facilities, by treating the malaria cases and referring those cases not considered as caused by malaria to the health facility level. This was possible since the training module of the CHWs/KOLs included recognition of the most prevalent non-malaria illnesses in the area (mainly cough with fast breathing proxy for pneumonia, diarrhoeal diseases) and the recommendation of immediate referral to the health system to avoid unnecessary delay in the management of those cases. Also, after two days of home treatment, persistence of the fever warrants a visit to the health facility, since this is more likely to be attributable to other causes than malaria. Unfortunately, no data were collected to document the possible indications for referral.

The feasibility and acceptability of the use of ACTs within the context of HMM has been demonstrated by Ajayi et al [13], in a multi-centre study involving four African sites. The present study confirms this finding, as reflected by the high proportion of malaria cases treated by CHWs/KOLs in the intervention arm. The level of knowledge of the CHWs/KOLs of the common clinical symptoms of malaria, and the accuracy in the prescription of study drug was appreciable at the post training survey. There is, therefore, a potential for well-trained CHWs/KOLs to increase the access of the population to appropriate treatment for malaria.

One of the concerns about the wide-scale use of ACTs by the CHWs/KOLs is the possibility of over-prescription of the drug, leading to wastage and possibly increasing the risk of emergence of resistance $[12,15,16]$. This cannot be excluded in this study. The use of rapid diagnosis tests to target the treatment of true malaria cases with the ACTs is one of the solutions being explored. It has the potential of adding great value to the CHWs/KOLs current performance in managing malaria close to the home, by avoiding the unnecessary delay in the treatment of non-malaria illness, which could be observed with the current presumptive treatment strategy.

The increase in the number of malaria cases treated at the community level is associated with a reduction of the overall workload at health facility level. In the study, the HF attendance rate was lowered by $43 \%$ in the intervention arm, with most malaria cases being treated in the community. In the breakdown of disease episodes treated 
at HF level, malaria accounts for $21 \%$ of all attendances in the HF of the intervention arm, while it represents $70.7 \%$ in the HF of the control arm. The implications of this finding are obvious.

In developing countries, where malaria burden poses a challenge to the health system, the first level of care for the sick patients is the one that usually bears the high burden of the disease. In this study, the CSPS represents the first point of contact of sick children with the health system, and the workload imposed by the treatment of malaria cases in children by health personnel is not negligible. The intervention has demonstrated the potential of reducing this burden by at least $70.0 \%$.

The health workforce shortage crisis in developing countries is one of the major challenges the health system is facing; it is estimated that to achieve even a modest coverage for essential health interventions, the health worker density required is 2.28 per 1,000 population[17]; in the context of the study area, the actual rates were respectively one nurse per 3,700 population and one doctor per 30,000 [18]. Shortages of health workers in sub-Saharan Africa derive from many causes; among others, one could include past investment shortfalls in pre-service training, morbidity and premature mortality, international migration, premature retirement $[19,20]$.

While various solutions are being explored by policymakers to circumvent this problem, an intervention reducing by $70 \%$ the workload imposed by the treatment of malaria cases in children, could be seen as intermediate solution for malaria endemic countries. Indeed, in the context of limited workforce, the precious time saved could be used by the health staff, for other curative or preventive activities, or to allow a longer nurse-patient contact time, a factor known to be associated with correct management of patients, and higher adherence by caregivers [21].

The home-management of other diseases, such as pneumonia and diarrhoea, is being investigated [22]. There is no doubt that if this could be achieved successfully, as what was observed for malaria, this might contribute to the Millennium Development Goal of reducing children mortality with the increase of the prompt access to appropriate treatment.

\section{Conclusion}

This study has shown that well-trained CHWs/KOLs could provide appropriate treatment for the majority of childhood malaria episodes in their respective communities, and thereby reduce the workload of health staff operating at HF level. This finding provides evidence that a scalingup of the HMM strategy might contribute to minimize the consequences of workforce shortage at peripheral health facilities level in most of the malaria endemic countries, and possibly improve their performance.

\section{Competing interests}

The authors declare that they have no competing interests.

\section{Authors' contributions}

SBS conceived the study and its design; he coordinated the data collection, the analysis and interpretation of the results and the review of the manuscript. ABT participated to the design of the study, the data collection and analysis and has drafted the manuscript. YK, AT and NC participated in the data collection and data analysis and interpretation. FP contributed in designing the study, monitoring the program implementation and revising the manuscript. All authors have read and approved the final manuscript.

\section{Acknowledgements}

We sincerely thank all the CHWs/KOLs who dedicated part of their time to this study; the caregivers who adopted this strategy. We also thank the health personnel in the district of Saponé, who helped in supervising the activities of the $\mathrm{CHWs} / \mathrm{KOLs}$.

This investigation received financial support from the UNDP/World Bank/ WHO Special Programme for Research and Training in Tropical Diseases (TDR) (Project ID number: A30930).

\section{References}

I. Rowe AK, Rowe SY, Snow RW, Korenromp EL, Schellenberg JRMA, Stein C, Nahlen BL, Bryce J, Black RE, Steketee RW: The burden of malaria mortality among African children in the year 2000. Int J Epidemiol 2006, 35:691-704.

2. Breman JG, Alilio MS, Mills A: Conquering the intolerable burden of malaria: what's new, what's needed: A summary. Am J Trop Med Hyg 2004, 7 I: I- I5.

3. Nahlen BL, Korenromp EL, Miller JM, Shibuya K: Malaria risk: Estimating clinical episodes of malaria. Nature 2005, 437:E3.

4. WHO: The African Summit on Roll Back Malaria, Abuja, Nigeria. WHO/CDS/RBM/2000I 725 April 2000.

5. WHO: The Roll Back Malaria strategy for improving access to treatment trough home management of malaria. WHOI HTM/MAL/2005 I I0I.

6. Kidane G, Morrow RH: Teaching mothers to provide home treatment of malaria in Tigray, Ethiopia: a randomised trial. Lancet 2000, 356:550-555.

7. Pagnoni F, Convelbo N, Tiendrebeogo J, Cousens S, Esposito F: A community-based programme to provide prompt and adequate treatment of presumptive malaria in children. Trans $R$ Soc Trop Med Hyg 1997, 91:512-517.

8. Sirima SB, Konate A, Tiono AB, Convelbo N, Cousens S, Pagnoni F: Early treatment of childhood fevers with pre-packaged antimalarial drugs in the home reduces severe malaria morbidity in Burkina Faso. Trop Med Int Health 2003, 8: I33-I 39.

9. Ibrahim ML, Hassane H, Konate L, Adamou S, Ousmane I, Adehossi E, Jeanne I, Duchemin JB: [Plasmodium falciparum chloroquine and pyrimethamine resistance monitoring network with molecular tools in the Niger River valley, Republic of Niger]. Bull Soc Pathol Exot 2008, I 0 I:47-49.

10. Meissner PE, Mandi G, Mockenhaupt FP, Witte S, Coulibaly B, Mansmann U, Frey C, Merkle H, Burhenne J, Walter-Sack I, Muller O: Marked differences in the prevalence of chloroquine resistance between urban and rural communities in Burkina Faso. Acta Trop 2008, 105:81-86. 
II. Tinto H, Guekoun L, Zongo I, Guiguemde RT, D'Alessandro U, Ouedraogo JB: Chloroquine-resistance molecular markers (Pfcrt T76 and Pfmdr-I Y86) and amodiaquine resistance in Burkina Faso. Trop Med Int Health 2008, 13:238-240.

12. D'Alessandro U, Talisuna A, Boelaert M: Editorial: Should artemisinin-based combination treatment be used in the homebased management of malaria? Trop Med Int Health 2005, 10:1-2.

13. Ajayi IO, Browne EN, Garshong B, Bateganya F, Yusuf B, Agyei-Baffour P, Doamekpor L, Balyeku A, Munguti K, Cousens S, Pagnoni F: Feasibility and acceptability of artemisinin-based combination therapy for the home management of malaria in four African sites. Malar J 2008, 7:6.

14. Chinbuah AM, Gyapong JO, Pagnoni F, Wellington EK, Gyapong M: Feasibility and acceptability of the use of artemether-lumefantrine in the home management of uncomplicated malaria in children 6-59 months old in Ghana. Trop Med Int Health 2006 II:1003-1016.

15. Muula AS, Rudatsikira E, Siziya S, Mataya RH: Estimated financial and human resources requirements for the treatment of malaria in Malawi. Malar J 2007, 6:168.

16. Pagnoni F, Kengeya-Kayondo J, Ridley R, Were W, Nafo-Traore F, Namboze J, Sirima S: Artemisinin-based combination treatment in home-based management of malaria. Trop Med Int Health 2005, 10:621-622.

17. Mohr J: Health workers: a global profile. The World Health Report 2006, Chapter I:.

18. MED: Enquête Démographique et de Santé. 2003:445.

19. Mullan F: The metrics of the physician brain drain. N EnglJ Med 2005, 353:|810-18|8.

20. Zurn P, Dolea C, Stilwell B: Nurse retention and recruitment: developing a motivated workforce. Geneva International Council of Nurses. [http://www.icn.ch/global/ Issue4Retention.pdf]. accessed 23 April 2008

21. Rowe AK, Hamel MJ, Flanders WD, Doutizanga R, Ndoyo J, Deming MS: Predictors of correct treatment of children with fever seen at outpatient health facilities in the Central African Republic. Am J Epidemiol 2000, I5 I:1029-1035.

22. Richards DA, Toop LJ, Epton MJ, McGeoch GR, Town GI, Wynn-Thomas SM, Dawson RD, Hlavac MC, Werno AM, Abernethy PD: Home management of mild to moderately severe communityacquired pneumonia: a randomised controlled trial. Med Aust 2005, 183:235-238.

Publish with Bio Med Central and every scientist can read your work free of charge

"BioMed Central will be the most significant development for disseminating the results of biomedical research in our lifetime. "

Sir Paul Nurse, Cancer Research UK

Your research papers will be:

- available free of charge to the entire biomedical community

- peer reviewed and published immediately upon acceptance

- cited in PubMed and archived on PubMed Central

- yours - you keep the copyright

Submit your manuscript here:

http://www.biomedcentral.com/info/publishing_adv.asp
BioMedcentral 